\title{
La territorialisation des politiques éducatives en
}

\section{France}

Des tensions récurrentes

The "territorialization" of educational policies in France. Some recurring

tensions

La territorialización de las políticas educativas en Francia. Unas tensiones

recurrentes

Daniel Frandji

\section{(2) OpenEdition}

\section{Journals}

Édition électronique

URL : https://journals.openedition.org/ries/6076

DOI : 10.4000/ries.6076

ISSN : 2261-4265

\section{Éditeur}

France Education international

\section{Édition imprimée}

Date de publication : 1 décembre 2017

Pagination : 115-124

ISBN : 978-2-85420-616-6

ISSN : 1254-4590

Référence électronique

Daniel Frandji, "La territorialisation des politiques éducatives en France », Revue internationale d'éducation de Sèvres [En ligne], 76 | décembre 2017, mis en ligne le 01 décembre 2019, consulté le 24 juin 2021. URL : http://journals.openedition.org/ries/6076 ; DOI : https://doi.org/10.4000/ries.6076 


\title{
La territorialisation des politiques éducatives en France
}

\section{Des tensions récurrentes}

\author{
Daniel Frandji \\ ESPE-Université Lyon 1, UMR Triangle
}

En France, la notion de territorialisation des politiques éducatives désigne un processus institutionnel hétérogène. Celui-ci a été amorcé au début des années quatre-vingt avec les premières lois de décentralisation et, surtout, la mise en place des zones d'éducation prioritaires (Zep), dont le premier objectif avait été de « contribuer à corriger l'inégalité sociale par le renforcement sélectif de l'action éducative dans les zones et les milieux sociaux où le taux d'échec scolaire est le plus élevé » (circulaire de 1981). Depuis lors, cette territorialisation se déploie par l'intermédiaire des différents " dispositifs » engageant des " partenariats » entre l'Éducation nationale, d'autres ministères et les collectivités territoriales. Ce processus est hétérogène en ce sens qu'il résulte de logiques différentes qui peuvent faire masse autour de thèmes communs (Charlot, 1994), certains génériques, d'autres plus spécifiques à la question éducative : critique de la standardisation nationale et des pesanteurs bureaucratiques, valorisation de l'échelle humaine ou de la démocratie de proximité, thématique de l'école "ouverte ", argument de la nécessaire adaptation de la politique éducative à l'hétérogénéité des territoires ou de ses "publics", etc. Mais en tant que tel, ce processus redessine, en permanence, les frontières spatiales, temporelles et symboliques de l'institution scolaire et, de fait, constitue l'une des principales orientations de l'action publique dont il convient de suivre les développements en rapport avec les dynamiques de la fragmentation de l'offre éducative telle qu'elle s'observe, aussi, dans ce pays.

C'est ainsi que l'on peut questionner deux des récentes mesures qui s'inscrivent dans cette démarche. La première concerne justement cette politique d'éducation prioritaire (EP) que l'action gouvernementale s'est attachée à "refonder ». La seconde est celle qui s'est énoncée comme réforme des rythmes scolaires, assortie de la généralisation des projets éducatifs de territoire (PEDT). Dans le cadre de cet article, il s'agira toutefois moins de décrire en profondeur l'action engagée dans l'une et l'autre de ces mesures, que de relever les philosophies très différentes qu'elles peuvent incarner, même si celles-ci procèdent d'une commune politique gouvernementale. 
C'est que ces deux mesures ont conjointement été portées par la loi du 8 juillet 2013, dite aussi loi de "refondation de l'École de la République ", dont le principal objectif a été de lancer une série de réformes coordonnées visant à « réduire les inégalités et favoriser la réussite de tous " ${ }^{1}$. Il est vrai que la situation de l'institution scolaire française exige une action forte en la matière : tous les indicateurs nationaux et internationaux montrent comment ces inégalités se sont accrues depuis le début des années 2000, jusqu'à placer la France en tête "des pays de l'OCDE où l'origine sociale des enfants pèse le plus lourd dans les résultats scolaires $»^{2}$. Dans ses textes d'accompagnement, la loi de refondation se donne pour objectif «d'inverser la tendance ». Le nom qui donne son nom à la loi exprime d'ailleurs autant l'ambition que la particularité de l'entreprise. Il s'agit donc de "Refonder l'École de la République », et " [r] efonder ne signifie pas refondre à partir d'une tabula rasa, mais réexaminer pour donner du sens en se ressourçant sur des valeurs", ou encore : "ni se contenter d'aménager l'existant ni de mettre à bas l'édifice ${ }^{3}$. Les objectifs poursuivis par la loi sont néanmoins pluriels, à la hauteur des 26 mesures initialement impulsées.

\section{ÉCOLE À PLUSIEURS VITESSES ET REFONDATION DE L'ÉDUCATION PRIORITAIRE}

Le récent rapport Grande pauvreté et réussite scolaire prolonge les données statistiques disponibles sur l'aggravation des inégalités de performance scolaire en France (Delahaye, 2015). Il insiste sur ce que cela peut signifier historiquement et tire un signal d'alarme supplémentaire. De fait, quarante ans après l'installation du collège unique ou ce qui en tient lieu, il serait, sur ces bases, tout simplement impossible de parler « de système éducatif au singulier dans un pays où les écarts sont devenus aussi importants» (ibid). Le signal d'alarme est précisément lié au constat du devenir de plus en plus fragmenté du système scolaire français, qui, de socialement sélectif, semble devenir de plus en plus ségrégatif, jusqu'à donner l'impression

[d'un] formidable retour en arrière en matière d'organisation scolaire avec la quasi-reconstitution d'un système éducatif d'avant la démocratisation de l'accès aux études secondaires, fonctionnant en réseaux d'établissements juxtaposés selon les catégories sociales ou en filières différenciées au sein même des établissements. (idem.)

Bien sûr, l'argument du "retour en arrière » est insatisfaisant, comme d'ailleurs la lecture complète du rapport permet de le reconnaître. Il ne tient pas compte de la différence de situation, tant du point de vue des types d'organisation

1. Sous la précédente présidence de François Hollande. [https://goo.gl/WmbyP8]

2. [https://goo.gl/EuwEmr]

3. Dans les termes du rapport de la concertation préalable à la loi (p. 3) : [https://goo.gl/wGjdd1] 
scolaire ainsi rapprochés, que du point de vue de ce qu'elles engagent, impliquent et signifient dans leurs contextes historiques et sociaux réciproques. Mais il faut bien comprendre que l'analyse ici tenue se déploie dans la problématique des inégalités sociales, et qu'ici, du moins pouvons-nous ainsi l'argumenter, la problématique de la fragmentation, observée et dénoncée, se conçoit comme problème de la (ré)émergence d'une école à plusieurs vitesses.

Ce rapport s'inscrit assurément dans les dynamiques créées par la loi de refondation (Jean-Paul Delahaye, qui a coordonné sa rédaction, a été l'un des principaux artisans de la loi). Il introduit cependant des précisions notables dans le "diagnostic", qui conduisent à infléchir quelques problématisations habituelles des inégalités scolaires. La première précision est d'ailleurs la plus fondamentale en ce qu'elle implique un changement global de raisonnement : il faut cesser de concevoir l'échec scolaire des plus pauvres comme un "accident»:

Celui-ci est « inhérent à un système qui a globalement conservé la structure et l'organisation adaptées à la mission qui lui a été assignée à l'origine : trier et sélectionner.

Sans oublier que :

Les dysfonctionnements de notre école qui ne parvient pas à réduire les inégalités ne nuisent pas à tout le monde. La méritocratie a une face claire, pour ceux qui en bénéficient, et une face sombre pour tous les autres.

D'où la seconde précision visant sans doute à argumenter la nécessité de revenir sur cette mission assignée à l'école, voire à renouveler certaines des attentes de "justice» investies en cette unique figure méritocratique :

À ce niveau atteint par les inégalités, il devient absurde et cynique de parler d'égalité des chances, c'est à l'égalité des droits qu'il faut travailler.

Mais d'où encore cette troisième précision :

l'accent sur la grande pauvreté et les inégalités sociales ne doit pas conduire à (...) exonérer l'école de ses propres responsabilités.

Et, s'il convient de rappeler que "refonder l'école ", c'est " corriger les inégalités ", donc "faire réussir les plus pauvres" (ibid.), il convient surtout d'être particulièrement attentif au fait que

toutes les organisations pédagogiques ne se valent pas (...). Ces faits ne sont plus de l'ordre de l'opinion, ils sont établis par la recherche en éducation" (ibid.).

Ces précisions sont bien sûr susceptibles d'interprétations variées et mériteraient de plus longs développements. Mais, à l'instar des travaux de recherches en sciences sociales sur lesquels elles s'appuient, celles-ci engagent les prémisses d'une problématisation que l'on pourrait dire relationnelle et dynamique des inégalités scolaires. Les inégalités sont mises en relation avec le durcissement de la compétition scolaire soutenue par le fonctionnement hyper sélectif du système scolaire, tel que construit dès le début des années soixante, en lien avec les besoins de l'investissement économique, et au nom du principe 
méritocratique. Il s'agit dans le même temps de rappeler en quoi la société française n'a pas achevé la "démocratisation » de ce système, en ne menant pas à son terme l'institution du « collège unique » (décrété en 1975), notamment du point de vue pédagogique. Et, bien plus que dans d'autres réformes portées par la loi, c'est assurément dans le cadre de l'entreprise dite de refondation de la politique d'éducation prioritaire que cette problématisation a semblé être la plus opérante ${ }^{4}$.

L'action ainsi engagée semble en tout cas tirer des leçons des différents problèmes qui ont accompagné le développement de cette politique d'éducation prioritaire (EP). À bien des égards, celle-ci est de toute façon emblématique des enjeux, de l'hétérogénéité, et des ambiguïtés du processus de territorialisation dans le domaine éducatif, qu'elle a d'ailleurs contribué à initier avec les Zep de 1981. C'est ainsi que l'analyse des évolutions de cette politique, en France, comme dans d'autres pays européens, montre comment s'y succèdent et s'y entremêlent trois âges ou modèles porteurs de trois approches politiques très différentes (Demeuse, Frandji, Greger, Rochex, 2011).

Dans son premier âge, la politique d'EP semblait vouloir combiner une approche compensatoire de renforcement sélectif de l'action en territoires défavorisés avec la rénovation de l'ensemble du système pour lutter contre l'inégalité sociale. L'hypothèse est alors faite que, par propagation, l'EP puisse entraîner la réforme de l'ensemble du système ou, à tout le moins, compenser les difficultés spécifiques qui pèsent sur certains territoires (Rochex, 2016). On sait comment cette intentio auctoris a été fortement limitée par ses intentio operis : notamment les conceptions déficitaristes travaillant les analyses et les pratiques déployées. Elle l'a été par la faiblesse des "moyens supplémentaires » finalement attribués aux zones ciblées, ainsi que par l'absence d'outillage de la mise en œuvre, laissée à l'initiative des équipes locales enjointes, seules, d' " innover ». Le second modèle d'EP qui se déploie au cours des années 1990 cherche moins à lutter contre les inégalités sociales en matière de scolarisation qu'à garantir, dans les territoires propices à "l'exclusion ", l'accès à un seuil minimal de connaissances et de compétences. L'action politique se montre moins soucieuse du fonctionnement sélectif du système scolaire que du sort de ses "perdants" et des problèmes de "violence ", "d'absentéisme » et de "décrochage scolaire ». Au cours des années 2000, les inflexions de l'EP dessinent un troisième modèle, orienté vers la prise en charge de toutes sortes de catégories de populations perçues comme exposées à des "risques " spécifiques de ne pouvoir donner la pleine mesure de leurs «talents », de leurs " potentiels », ou de leur " excellence propre ». Ces trois approches se nourrissent de modes de ciblage différents (l'ensemble des élèves dans les établissements qui concentrent les difficultés, les élèves les plus en difficulté et ceux considérés dans la diversité de leurs caractéristiques individuelles, voire naturelles). Elles ne relèvent pas des mêmes conceptions de la justice ni des mêmes objectifs. Elles marquent le recul de la référence aux questionnements

4. Pour les grandes lignes de présentation de cette réforme : [https://goo.gl/Rccdoz] 
de la sociologie critique (en partie mobilisée dans le premier âge), au profit d'approches individualisantes voire essentialisantes ${ }^{5}$. Elles peuvent certes permettre de dénoncer un renoncement politique aux visées de démocratisation et de lutte contre les inégalités investies à partir des années soixante. Mais elles accompagnent aussi une forme de réactualisation exacerbée de la conception individualiste de l'égalité "méritocratique", laquelle oblige de plus en plus à devoir "réguler» les tensions sociales induites par les "missions de tri et de sélection » ainsi assignées au système scolaire.

$\mathrm{Du}$ reste, il est possible de mettre en relation les dernières orientations de la politique d'EP - celles d'avant la refondation - avec le détail de l'analyse statistique de l'aggravation des inégalités de performance des élèves. L'un des maîtres mots de ces orientations a été la promotion des "élèves méritants". Celle-ci s'est déclinée dans de nombreux dispositifs prenant le risque de renforcer une vision "mortifère » des quartiers populaires et de leurs établissements (par exfiltration de ces " méritants " visant à élargir leur accès à « l'excellence »). Elle s'est accompagnée de la mise en place d'un minimum curriculaire. Et elle a été assortie de la montée d'autres "dispositifs» déclinant bien plus souvent une conception sociale que scolaire de la "réussite éducative ». Ces dispositifs sont d'ailleurs moins pilotés par le ministère de l'éducation nationale que par celui de la ville, ou de la cohésion sociale, et sont de plus en plus pris en charge par les collectivités locales. Or, si les inégalités scolaires d'origine sociale se sont accrues depuis le début du XXI ${ }^{e}$ siècle, c'est d'abord parce que les résultats des élèves des milieux les plus défavorisés se sont dégradés, mais aussi parce que, plus récemment, les élèves favorisés ont vu leurs compétences progresser ${ }^{6}$.

S'il est trop tôt pour en apprécier les effets, les transformations à nouveau impulsées par la "refondation» de l'EP paraissent alors clairement à rebours des précédentes. On peut dire de la particularité de cette action réformatrice qu'elle a voulu renouer avec «l'approche compensatrice-transformatrice " caractérisant la première génération de la politique d'EP, tout en prenant en considération les travaux qui ont montré qu'il ne suffit pas « d'innover pour démocratiser le système éducatif et réduire les inégalités sociales» (Rochex, 2016). Ceci tient d'abord au fait d'avoir proposé, pour la première fois, un travail d'instrumentation de la réflexion et de l'action des acteurs professionnels de l'EP, notamment sous la forme d'un Référentiel pour l'éducation prioritaire. Cette démarche est originale en ce qu'elle se donne les moyens d'échapper à ce qui apparaît comme une sorte de double écueil «de l'appel à l'innovation d'une part, ou, d'autre part, de la prescription de "bonnes pratiques" standardisées" (idem). Elle l'est autant en fonction des modalités de travail ainsi promues, outillant les acteurs professionnels en matière d'analyse critique des processus de production de l'inégalité scolaire,

5. Nous empruntons largement ici les reformulations condensées de l'étude mentionnée, telles qu'effectuées par Rochex, 2016.

6. Comme le détaille le rapport sur les inégalités du CNESCO de 2016 : [https://goo.gl/PjBDhF] 
favorisant la mise en œuvre d'actions moins socialement privilégiantes. Mais de plus, pour éviter les effets de zonage, et renforcer la visée "transformatrice", la refondation de l'EP a aussi voulu se concrétiser (au moins en principe) par la mise en œuvre d'un principe d'allocation différentielle des moyens appliqué à l'ensemble des établissements - dont la dotation serait proportionnelle à l'ampleur des difficultés sociales et scolaires de leurs élèves. "Ni se contenter d'aménager l'existant, ni mettre à bas l'édifice »: l'étrange formule pourrait en effet ici paraître moins abstraite.

\section{LA GÉNÉRALISATION DES PROJETS ÉDUCATIFS DE TERRITOIRE}

La « réforme des rythmes scolaires », assortie de la généralisation des projets éducatifs de territoire (PEDT) instituée par la même loi, relève d'une autre conception de la territorialisation. On relève d'abord la trajectoire complexe de cette réforme, dont les contours et les enjeux se sont progressivement transformés sous l'effet des «solutions " retenues visant à résoudre les problèmes techniques d'organisation et de financement assurant sa mise en ouvre (Frandji et Morel, 2017). Celle-ci avait initialement pour objectif de résoudre un problème d'organisation du temps scolaire mis en avant comme délétère pour les élèves : la très grande concentration du temps de l'enseignement primaire en France, déjà ancienne, mais renforcée, depuis 2008, par la mise en place de la semaine de quatre jours seulement de classe, de six heures d'enseignement chacun, pour un total de 144 jours par an (en dessous de la moyenne dans les pays de l'OCDE). La réforme essaie de déconcentrer ces temps, en faisant de "l'intérêt des élèves sa priorité absolue». Elle encadre différentes possibilités d'organisation, réglementant le nombre de jours hebdomadaires (qui doit être supérieur), celui des heures journalières (qui doit être inférieur), ainsi que le temps de la pause méridienne (qui doit être supérieur). Mais ces règles, contestées par des élus locaux et des parents, d'autant plus écoutés qu'ils sont aussi des électeurs, vont progressivement être assouplies, par le jeu de dérogations, ou de droit à " l'expérimentation locale ». Et, surtout, la particularité de la réforme est de confier la gestion de ces «temps libérés » ou réaménagés aux municipalités qui doivent mettre en place des «projets éducatifs » (avec appel à la mobilisation de la société civile, dans une visée participative). Ceux-ci, les PEDT, formalisent

une démarche permettant aux collectivités territoriales volontaires de proposer à chaque enfant un parcours éducatif cohérent et de qualité avant, pendant et après l'école $[\ldots]$.

Ils doivent assurer la «complémentarité » et la «continuité » des temps éducatifs (les maîtres mots de la réforme) ; mais aussi comme ensuite précisé, être un instrument « souple et adaptable à toutes les réalités locales » (circulaires respectives de mars 2013, puis de décembre 2014). Ils prennent la forme d'un 
contrat dont la signature conditionne une aide financière de l'État, visant à réduire les coûts occasionnés par le changement d'organisation pour les collectivités. C'est donc la double nature et l'ambition de cette réforme des rythmes scolaires, que de susciter des changements locaux dans les manières de conduire les politiques éducatives, de vouloir faire bouger le territoire éducatif global, autant que de remodeler les emplois du temps.

D’une part, cette réforme conduit à quelque peu réviser ce qui avait déjà pu être observé à propos du processus historique de territorialisation de l'action éducative. Au début des années quatre-vingt-dix, ce processus avait été décrit comme une "politique nationale ", voulue, définie et organisée par l'État, et nullement comme " une conquête du local " (Charlot, 1994). Or, la mise en place des PEDT prend modèle sur des projets expérimentés depuis un moment déjà dans certaines collectivités, qui s'en sont fait d'ardents promoteurs. Et la réforme dans son ensemble porte la marque des réseaux d'acteurs qui ont ensemble beaucoup œuvré pour la reconnaissance de ces expériences dans la loi, même s'ils ne partagent pas toujours la même conception de l'éducatif (réseaux regroupant notamment une association d'élus locaux souhaitant installer le label de «villes éducatrices »; un regroupement des « nouveaux » professionnels, qui, depuis une vingtaine d'années, assurent la coordination du travail éducatif dans les collectivités ; ainsi que des grandes fédérations d'éducation populaire, qui forment les acteurs de l'animation et gèrent des structures péri et extra-scolaires).

D’autre part, surtout, les dynamiques créées par la réforme apparaissent plurielles, complexes, ambivalentes. C'est du moins ce qui a été observé par les quelques études empiriques sérieuses qui se sont aventurées sur ce terrain (pour une présentation de ces travaux, voir Frandji et Morel, 2017). Plusieurs observations sont ici mobilisables. La première montre une limite notoire de la réforme, telle qu'elle s'est faite. Dans l'absolu (on peut ainsi le concevoir), le déploiement des PEDT est susceptible d'enrichir la réflexion entretenue par l'organisation des temps scolaires et éducatifs, souvent réduite à des considérations expertes "chronobiologisantes» sur la "fatigue» des enfants, ou leurs "pics de vigilance ", ne tenant pas compte des contenus et des activités qui leurs sont proposés. Il pourrait d'ailleurs même obliger à ne plus se contenter de simplement faire référence à "l'intérêt des élèves " comme "priorité absolue ", sans se préoccuper de préciser la manière dont sont définis ces «intérêts » : par qui, pourquoi, comment, et, surtout, de quels élèves? Mais, sur le terrain, on observe comment la mise en place organisationnelle des PEDT accapare les acteurs des territoires, aux prises avec des contraintes gestionnaires, et de nombreux problèmes "techniques", remettant toujours à plus tard le travail sur ses contenus ou comme ils le disent, son "sens ». De plus, la réforme implique de nouvelles articulations entre préoccupations nationales et locales, qui ellesmêmes monopolisent la réflexivité nationale sur les risques de disparités, voire de nouvelles inégalités territoriales et géographiques, au détriment des problématiques liées aux inégalités sociales en matière d'éducation. 
Deuxièmement, on observe comment l'appel à la mobilisation de la «société civile », qui accompagne la fabrique des PEDT, et la multiplication des professionnels intervenant auprès des enfants, activent une variété d'attentes et de discours éducatifs qui tendent à minimiser la spécificité des apprentissages scolaires pour le développement cognitif et social des enfants. En ce domaine, des " contextes » semblent s'accompagner de différences : contexte social des publics et usagers dont on doit organiser les activités éducatives de leurs enfants, mais contexte urbain/rural aussi. Car les élus et acteurs des services municipaux n'accordent pas la même confiance à l'école ni à ses professionnels. S'ils sont d'un milieu rural, ils semblent vouloir respecter, garantir et/ou renforcer le « territoire d'activité » spécifique de l'école, tandis qu'ils semblent bien plus souvent vouloir le relativiser, le redéfinir ou le transformer en milieu urbain. Il est vrai que les discours en milieu urbain populaire sont aussi les plus marqués par les catégories des politiques de la ville et/ou des problématiques éducatives déjà éprouvées caractérisant "l'âge des dispositifs" (Barrère, 2013). En fait, si bien des acteurs des collectivités et de l'animation n'entendent surtout pas "faire l'école après l'école »-nonobstant la pression, dite surtout effectuée par certains milieux sociaux, à vouloir enrôler les activités éducatives "complémentaires" dans la course à la performance -, il est très rare de voir ces mêmes activités pensées autrement que dans la visée d'un modèle supplétif. L'apport potentiel de ces dernières, du point de vue éducatif et en matière de justice sociale, est alors limité, classiquement clôturé par l'idéologie légitimiste de la déficience culturelle (modèle compensatoire), des rhétoriques "spontanéistes », et une idéologie de la reconnaissance des différences à tonalité relativiste et psychologisante. En même temps, des observations montrent comment d'autres pratiques peuvent en ce domaine se chercher et s'expérimenter sur le terrain avec l'aide des mouvements d'éducation populaire. Ces recherches se déclenchent d'autant plus quand la "fabrique » des PEDT n'est pas totalement clôturée par les considérations gestionnaires et la pression de l'urgence, qui voient tous les discours, les analyses et les pratiques, se standardiser dans le registre des orientations déjà instituées des « dispositifs».

Enfin le dernier constat conduit à renouveler les problématiques convenues de " complémentarité » et de " continuité éducative ", en s'attachant à questionner ce qui se joue du point de vue des enfants et de leurs apprentissages. L'analyse réalisée par Netter (2016) est riche de ce point de vue. Celle-ci met en lumière la progression de l'hétérogénéité des registres cognitifs d'activité auxquels sont confrontés les élèves, pour souligner la "grande segmentation » des divers temps éducatifs que ceux-ci sont de plus en plus conduits à vivre dans les écoles observées. Cette hétérogénéité, insistons-y, s'observe déjà dans chacun des temps " éducatifs » concernés, y compris les " temps scolaires " (comme " péri-scolaires " et d'intervention médico-sociale). En ce sens, comme d'autres travaux l'ont déjà montré, cette hétérogénéité n'est pas née avec la réforme des rythmes. Et on pourrait d'ailleurs même imaginer la possibilité de voir cette réforme contribuer à la questionner, sur le terrain. Pourtant, c'est l'inverse qui est observé, et J. Netter 
relève comment le temps de présence scolaire de certains élèves (plus encore ceux considérés en difficulté) se réalise en présence d'un toujours plus grand nombre d'intervenants divers, tout en étant implicitement composés d'une pluralité de segments d'activités hétérogènes,

régis par des logiques thématiques ou disciplinaires, orientés vers les apprentissages ou le jeu, privilégiant des systèmes de signes spécifiques et encadrés par des acteurs divers (Ibid.).

Or, si une telle segmentation peut profiter à certains élèves, les «plus performants » du point de vue scolaire, en ce qu'elle leur permet de développer des compétences de circulation entre des mondes sociaux pluriels ou de répondre de manière créative à un volume croissant de nouvelles expériences, ce n'est pas le cas pour d'autres. De fait, en l'absence d'un travail visant, dans le monde scolaire, à faciliter l'intégration des différents registres d'activité, seuls ceux qui bénéficient d'un tel travail de "couture " réalisé à la maison en tirent profit. Les autres ne parviennent pas à faire la synthèse de leur expérience, pour la transformer en "apprentissages ». Pour eux, la multiplication des temps opérée par la réforme des rythmes semble même être synonyme de difficultés accrues.

Aucune généralisation de ces observations ne peut être faite à ce stade, même si celles-ci sont corroborées par plusieurs travaux. Elles nous montrent toutefois en quoi les deux réformes considérées dans cet article, bien que conduites par le même gouvernement, ne relèvent pas de la même philosophie. D’une part, elles paraissent concéder des rôles différents à l'État : d'un État qui cherche à se réaffirmer comme "éducateur " (Charlot, 1994), à un État acteur parmi d'autres d'une forme de gouvernance multi-régulatrice (Maroy, 2007) ? Le premier est particulièrement concerné par les contenus de l'action publique éducative et tient compte de l'éclairage des sciences sociales qu'il s'attache à mieux mobiliser. Il cherche à renouveler ses formes d'intervention dans la perspective d'un "mode de pilotage pédagogique ", sans verser dans une logique managériale prescriptive de «bonnes pratiques » standardisées. Le second n'a certes pas renoncé à orienter et à contrôler l'action publique éducative mais il le fait sans guère de préoccupation sur ses contenus et orientations, qu'il délègue à tout un secteur de la consultance. Et il le fait par l'intermédiaire de nouveaux dispositifs d'incitation, d'accompagnement technique et, comme il se doit en théorie, "d'évaluation ». Mais les deux premiers s'en tiennent majoritairement à des questions techniques d'organisation, augmentées de quelques considérations légales ${ }^{7}$. Quant aux dispositifs d'évaluation, ou à ce qui se présente ainsi, ceux-ci favorisent l'emprise de nouveaux experts en éducation souvent rétifs aux questions des sciences sociales: dans les faits ces évaluations relèvent de critères d'appréciation de la réforme et des PEDT (de sa "qualité ») très divers. Ils se donnent notamment peu de possibilités de déplacer les questionnements portés par les débats très polarisés sur la réforme des rythmes (Frandji et Morel, 2017).

7. Voir sur ce point l'analyse de l'accompagnement numérique aux collectivités proposé au début de la réforme par l'intermédiaire d'un site internet gouvernemental : Frandji et al., 2015 
D'autre part, l'analyse montre surtout comment les deux réformes considérées n'incarnent pas les mêmes conceptions de ce qu'il est juste et bon de faire en matière d'éducation, voire contribuent à accentuer, en mode additif, plutôt qu'à dépasser, la tension récurrente entretenue par le processus de territorialisation des politiques éducatives initié au début des années quatre-vingts. Entre reprise de la lutte contre les inégalités sociales d'apprentissage, visant à conjurer la fragmentation induite par la dégradation sélective des contextes de scolarisation réservés aux enfants les plus défavorisés, et énième politique de régulation des tensions sociales générées par la constitution d'un système scolaire hyper sélectif et concurrentiel. Relance du processus de démocratisation de l'institution dans un cas, qui ne peut plus continuer à faire l'impasse sur les biais sociaux agissant dans les "modalités » de ses formes d'organisation, de ses pratiques et de ses hypothèses. Décrispation de la "réussite scolaire " dans l'autre, par référence à une rhétorique de "réussite éducative" bien floue se donnant pour objectif de contribuer au "développement harmonieux des enfants ${ }^{8}$, tout en prenant le risque d'accentuer une dynamique de segmentation de l'action éducative socialement privilégiante.

\section{BIBLIOGRAPHIE}

BARRÈRE A. (2013) : «La montée des dispositifs : un nouvel âge de l'organisation scolaire » in Carrefours de l'éducation, 2, n 36, p. 95-116.

CHARLOT B. (1994): L'école et le territoire. Nouveaux espaces, nouveaux enjeux, Paris : Armand Colin.

DELAHAYE J.-P. (2015) : Grande pauvreté et réussite scolaire. Le choix de la solidarité pour la réussite de tous, rapport à madame la ministre de l'Éducation nationale, de l'Enseignement supérieur et de la Recherche, Paris, IGEN, 2015.

DEMEUSE M., FRANDJI D., GREGER D., ROCHEX J.Y. (2011): Les politiques d'éducation prioritaire en Europe. Quel devenir pour l'égalité scolaire?, vol 2. Lyon: ENS éditions.

FRANDJI D. (dir), DOUCHY M., FOUNEL Y., MOREL R. et RANCON S. (2015) : Le projet éducatif de territoire (PEDT) : ses chiffres, ses mots, son rapport au monde social, en l'étape de sa généralisation, ENS de Lyon, IFE, en ligne: [https://goo.gl/MNYQbL]

FRANDJI D. et MOREL R. (2017) : La réforme des rythmes scolaires et les projets éducatifs de territoire: première analyse des évaluations, bilans et autres expertises réalisés entre 2013 et 2017, Paris : CNESCO, en ligne : [https://goo.gl/E7dYN8]

MAROY C. (2007) : «Les modes de régulation de l'École», Revue internationale d'éducation de Sèvres, 46, p. 87-98.

NETTER J. (2015) : La division du travail scolaire Segmentation, solidarité et inégalités dans l'école primaire contemporaine, thèse de doctorat en sciences de l'éducation, université Paris 8 Saint-Denis.

ROCHEX J.Y. (2016) : "Faut-il crier haro sur l'éducation prioritaire? Analyses et controverses sur une politique incertaine ", Revue française de pédagogie, 194, p. 91-108.

8. [https://goo.gl/3iiK1h] 